\title{
Sudden Intraventricular Conduction System Defect Prior to Urgent Coronary Artery Revascularization
}

\author{
Amer Harky ${ }^{1}$, Mohamad Bashir ${ }^{1 *}$, Martin Lees $^{2}$ and Neil Roberts ${ }^{1}$ \\ ${ }^{1}$ Department of Cardiac Surgery, Barts Heart Centre, St. Bartholomew's Hospital, London, UK \\ ${ }^{2}$ Department of Perioperative medicine, Barts Heart Centre, St. Bartholomew's Hospital, London, UK
}

Submission: February 01, 2017; Published: March 07, 2017

*Corresponding author: Mohamad Bashir, Barts Heart Centre, St. Bartholomew’s Hospital, London, UK, Email: drmbashir@mail.com

\begin{abstract}
Intraventricular conduction abnormalities have been thoroughly described in the setting of post cardiac surgery in particular after valvular surgery. It's also widely known that several anaesthetic factors are known to cause autonomic disturbances resulting in unmasking of a sinus node dysfunction, significant bradycardia, and cardiovascular collapse during the intraoperative period. However, little is known on asymptomatic episodes in particular prior to CABG. We report a rare occurrence of intraventricular conduction defect that presented in an asymptomatic patient following NSTEMI and prior to urgent coronary artery revascularization. We aim to highlight the measures that were taken to manage this.

Keywords: Conduction defect; Sick-sinus syndrome; Coronary revascularization

Abbrevations: NSTEMI: Non-ST Elevation Myocardial Infarction; ECG: Electrocardiogram; LCM: Left Main Stem; LAD: Left Anterior Descending Artery LCx: Left Circumflex RCA: Right Coronary Artery; CABG: Coronary Artery Bypass Grafting Surgery; AV: Atrioventricular CPB: Cardiopulmonary Bypass; LIMA: Left Internal Mammary Artery SVG: Saphenous Venous Graft; PDA: Posterior Descending Artery; AF: Atrial Fibrillation; TED: Transoesophageal Echocardiography; PPM: Permanent Pacemaker; VCD: Ventricular Conduction Defects
\end{abstract}

\section{Case Report}

We report a 61-year-oldmale, who was admitted to our centre with sudden onset of chest pain. He was diagnosed with non-ST elevation myocardial infarction (NSTEMI). His electrocardiogram (ECG) showed normal sinus rhythm with inferiolateral ST segment depression (Figure 1A). Patients past medical history include ex-smoker, controlled hypertension, non-insulin dependent diabetes mellitus, peripheral vascular disease, hypercholesterolemia, iron deficiency anaemia and Hepatitis B.

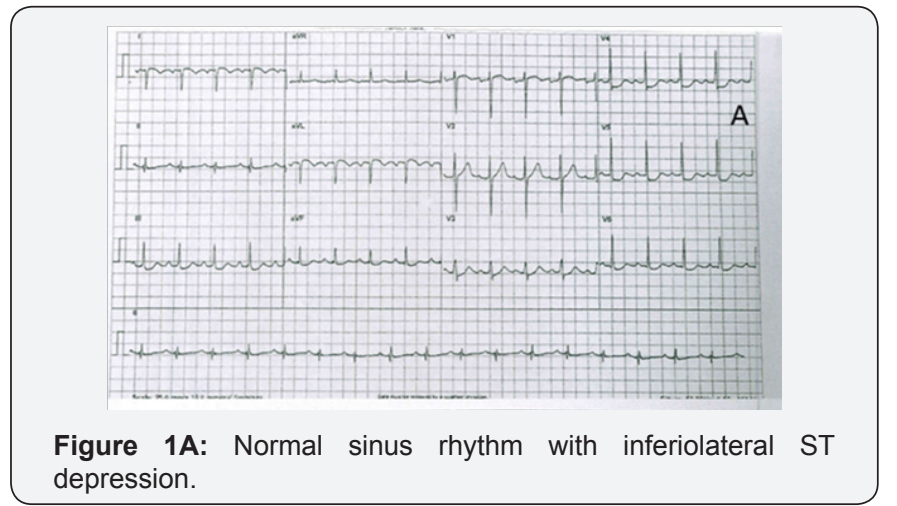

The patient's immediate angiogram revealed a right dominant heart with severe distal left main stem (LMS) disease which was $80 \%$ occluded. The left anterior descending artery (LAD) was diffusely irregular with severe mid-vessel disease. It was noted that the patient had a high diagonal artery with severely diseased proximal portion. The left circumflex (LCx) artery territory was with mild irregularities and severe diffuse disease. The right coronary artery (RCA) was diffusely diseased.

Patients' condition was optimized and stabilized medically. Due to diffuse multi-vessel involvement, the patient was deemed suitable for in-patient coronary artery bypass grafting surgery (CABG).

Seven days following his presentation, the patient underwent surgery. On induction of anaesthesia, whilst inserting the central venous line into right internal jugular vein, the patient develop edacute episode of sinus tachycardia followed by sinus bradycardia of 40 beats per minute. A bolus dose of $5 \mathrm{mcg}$ of adrenaline was given to maintain his heart rate. Preliminary impression was that patient must have sustained an infraction entailing the atrioventricular (AV) conduction system. 
Cardiopulmonary bypass (CPB) was instituted immediately and patient's status was stabilized.

He underwent CABG utilizing left internal mammary artery (LIMA) to LAD and saphenous venous graft (SVG) to obtuse marginal and posterior descending artery (PDA). The CPB time was $77 \mathrm{mins}$ and aortic cross clamp time was $35 \mathrm{mins}$. The patient was weaned off CPB support in sinus bradycardia. Two epicardial pacing wires were placed and patient was externally paced.

Three days following his CABG, patient became haemo dynamically unstable with alternate episodes of tachy-brady arrhythmias concomitant with atrial fibrillation (AF). For rate control, the patient was loaded with intravenous amiodarone. His status necessitated neither inotropic support with nor adrenaline to maintain adequate blood pressure and urine output.

Bedside transoesophageal echocardiography (TOE) was performed to exclude cardiac tamponade; the findings from the TOE were unequivocal with no presence of left atrial thrombus.

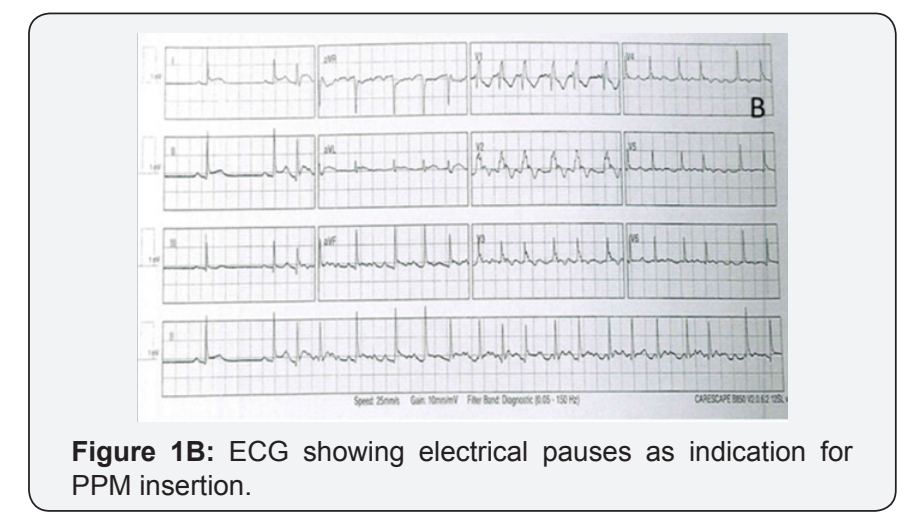

Subsequently, an urgent consultation with our local electrophysiology team was sought for. It was noted that the tachy-brady arrhythmias included multiple electrical pauses (Figure 1B) alternating with atrial fibrillation. The decision was made to insert a dual chamber permanent pacemaker (PPM). Following insertion of PPM, the patient was persistently sustaining intermittent rapid ventricular rate AF episodes and he was given oral beta-blocker. Following to this the patient's heart rate was controlled and he was eventually discharged to the ward.

\section{Discussion}

Electrical conduction abnormalities are well-recognized complications of acute myocardial infarction [1]. They are caused by either autonomic imbalance or ischemia and necrosis of the conduction system. The most common clinical consequence is bradycardia, which may or may not be symptomatic. Complete heart block with a slow escape rhythm is a potentially fatal event in this setting if not detected and treated promptly.

Anaesthesia related autonomic imbalance may unveil an undiagnosed sick sinus syndrome [2]. However, in diagnosed, asymptomatic patients one would contemplate preoperative insertion of a temporary pacemaker prior to anaesthetic induction as the latter had clearly been described altogether with surgical manoeuvres to induce an autonomic misbalance leading to serious dysrhythmias resistant to conventional pharmacological treatment.

Preoperative, intra operative and postoperative variables, which might play a role in the development of ventricular conduction defects (VCD) and atrial fibrillation (AF) following coronary artery bypass grafting (CABG), were previously described in the literature. Caretta et al. [3] evaluated 236 consecutive patients who sustained VCD and AF postoperatively and reported an incidence of $15.5 \%$ amongst their patients' cohort. In their analysis, they demonstrated that left main disease and right coronary artery occlusion associated with significant stenosis of the proximal left anterior descending artery were amongst the potential factor that could lead to conduction abnormalities. It also notable to mention that they reported on the duration of aortic cross-clamp time which was contributory factor to the development of conduction abnormalities and sequelae.

Compounded by these risks, it's quite common to practicing cardiac surgeons the knowledge of development of postoperative AF following cardiac surgery [4] and the link between ischaemic injuries, with increasing duration of cardioplegic arrest, seems to play a key role in such incidence. Yet, non homogeneous cardioplegic delivery to critical areas of myocardium, and particularly to the specialized conducting system, may cause VCD after CABG.

Passman et al. [5] described a model where by the incidence of AF post cardiac surgery could potentially be quantified and predicted. They describe the occurrence of conduction abnormalities to be related to advanced age $>65$ years, PR interval $>/=180$ milliseconds and a P-wave duration in lead V1 $>/=110$ milliseconds. Such peculiarities could potentially lead to prevention protocols to avert patients from sustaining AF post cardiac surgery which has long health and cost implications.

However, in this case report, we demonstrated that intra ventricular conduction defect and abnormalities occurred essentially at induction of anaesthesia in otherwise normal and asymptomatic patient with normal sinus preoperative ECG rhythm. Although bradycardia associated with vecuronium, alfentanil, and propofol were described as a potential contributing factors, however, pre-treatment with anti-cholinergic agents may reduce this incidence in healthy individuals, and hence bradycardia under anaesthesia shouldn't be assumed as iatrogenic as the cause might be sick sinus syndrome [6]. Bearing the following in mind, it was deemed necessary to expedite the patient to theatre and to commencing of CPB to avert further injury to the heart and avoid peri-arrest episodes.

Yet and despite the measures undertaken to correct the underlying ischaemic causes that potentially led to acute 
intraventricular stunning of the conduction system, the patient persistently continued to display signs of tachyarrhythmia and even after a PPM was sought for. This is a unique experience that opens the scope for further studies or models to predict occurrence of asymptomatic intraventricular conduction abnormalities prior to CABG and to overall correctable causes.

\section{Authors Contribution}

A.H. has prepared the first draft of the case report. M.B. has reviewed and made necessary changes in the case report. M.L. has reviewed the final manuscript and made changes. N.R. reviewed and approved final manuscript.

\section{References}

1. Trappe HJ (2010) Tachyarrhythmias, Bradyarrhythmias and Acute Coronary Syndromes. J Emerg Trauma Shock 3(2): 137-142.
2. Nag K, Nagella AB, Kumar VR, Singh DR, Ravishankar M (2015) Role of temporary pacing at the Right Ventricular Outflow Tract in Anesthetic Management of a Patient with Asymptomatic Sick Sinus Syndrome. Anesth Essays Res 9(3): 423-426.

3. Caretta Q, Mercanti CA, De Nardo D, Chiarotti F, Scibilia G, et al. (1991) Ventricular conduction defects and atrial fibrillation after coronary artery bypass grafting. Multivariate analysis of preoperative, intraoperative and postoperative variables. Eur Heart J 12(10): 11071111.

4. Alqahtani AA (2010) Atrial Fibrillation Post Cardiac Surgery Trends Toward Management. Heart Views 11(2): 57-63.

5. Passman R, Beshai J, Pavri B, Kimmel S (2001) Predicting PostCoronary Bypass Surgery Atrial Arrhythmias from the Preoperative Electrocardiogram. Am Heart J 142(5): 806-810.

6. Levy DM (1990) Recurrent Bradycardia due to Latent Sick Sinus Syndrome. Anaesthesia 45(6): 488-489.

\section{Your next submission with Juniper Publishers} will reach you the below assets

- Quality Editorial service

- Swift Peer Review

- Reprints availability

- E-prints Service

- Manuscript Podcast for convenient understanding

- Global attainment for your research

- Manuscript accessibility in different formats

( Pdf, E-pub, Full Text, Audio)

- Unceasing customer service

Track the below URL for one-step submission https://juniperpublishers.com/online-submission.php 